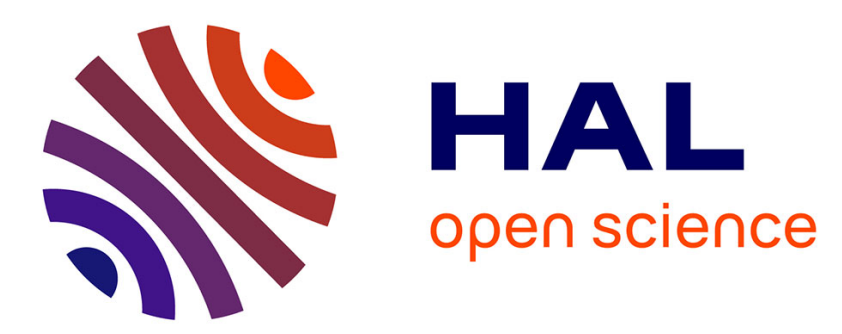

\title{
Optimal Static Pricing of Reverse-Link DS-CDMA Multiclass Traffic
}

\author{
Yezekael Hayel, Victor Ramos, Bruno Tuffin
}

\section{To cite this version:}

Yezekael Hayel, Victor Ramos, Bruno Tuffin. Optimal Static Pricing of Reverse-Link DS-CDMA Multiclass Traffic. [Research Report] PI 1731, 2005, pp.26. inria-00000151

\section{HAL Id: inria-00000151 \\ https://hal.inria.fr/inria-00000151}

Submitted on 7 Jul 2005

HAL is a multi-disciplinary open access archive for the deposit and dissemination of scientific research documents, whether they are published or not. The documents may come from teaching and research institutions in France or abroad, or from public or private research centers.
L'archive ouverte pluridisciplinaire HAL, est destinée au dépôt et à la diffusion de documents scientifiques de niveau recherche, publiés ou non, émanant des établissements d'enseignement et de recherche français ou étrangers, des laboratoires publics ou privés. 


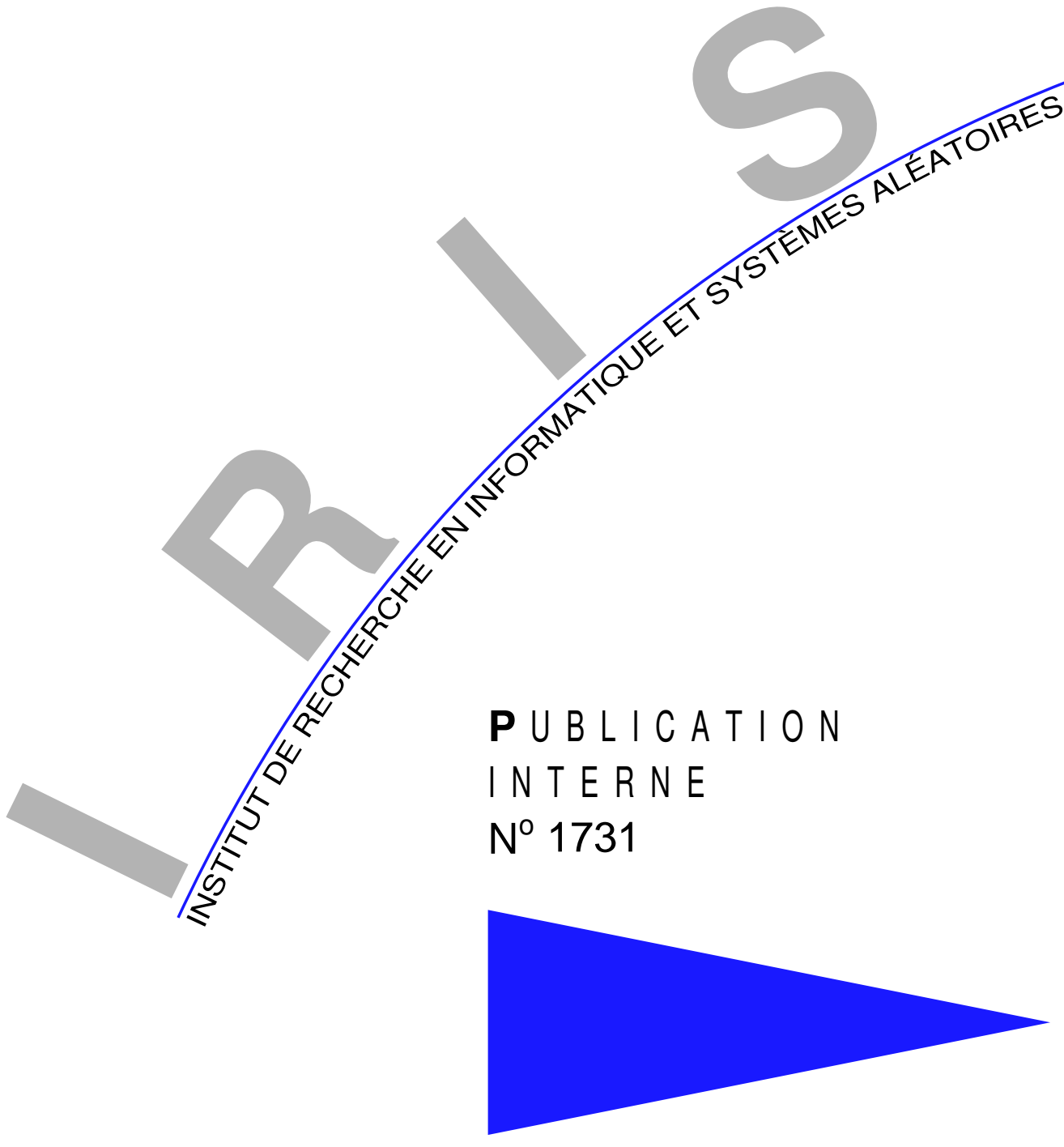

\title{
OPTIMAL STATIC PRICING OF REVERSE-LINK DS-CDMA MULTICLASS TRAFFIC
}

\author{
Y. HAYEL , V. RAMOS AND B. TUFFIN
}





\title{
Optimal Static Pricing of Reverse-Link DS-CDMA Multiclass Traffic
}

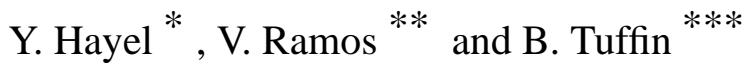 \\ Systèmes communicants \\ Projet Armor \\ Publication interne $\mathrm{n}^{\circ} 1731-$ Juillet $2005-26$ pages
}

\begin{abstract}
Third Generation (3G) wireless systems are expected to become more and more popular worldwide, thanks to a higher quality than in current $2 \mathrm{G}$. Direct-sequence code-division multipleaccess (DS-CDMA) is a solution to be implemented in these systems but, due to a limited radio spectrum and more and more demanding applications, it seems likely that congestion will still be a problem. Pricing appears a simple way to tackle this problem. This paper aims at studying the impact of a per-packet static pricing scheme on the use of the reverse-link in a cell where demand (defined through the so-called utility functions) decreases when prices increase or quality of service decreases. The paper also deals with the pricing of multiple classes, since DS-CDMA supports integrated services. In a first step, we determine as a Nash equilibrium the number of customers that will actually apply for service, depending on demand. In a second step, assuming perfect power control, we find the prices and received powers optimizing the service provider's revenue. We find that in the case where potential demand always exceeds capacity, the base station's best interest is to favor only one class, but that it is not the case in the more realistic situation when considering potential demand as a random variable over time.
\end{abstract}

Key-words: Pricing, Wireless CDMA Networks, Optimization

(Résumé : tsvp)

\footnotetext{
*yezekael.hayel@irisa.fr

** Victor.Ramos@ieee.org

****bruno.tuffin@irisa.fr
}

\section{Coss}

Centre National de la Recherche Scientifique (UMR 6074) Université de Rennes 1 - Insa de Rennes
Institut National de Recherche en Informatique et en Automatique - unité de recherche de Rennes 


\section{Tarification statique optimale de la voix montante dans une cellule DS-CDMA avec trafic hétérogène}

Résumé : Les systèmes de transmission sans fils de troisième génération $(3 G)$ deviennent de plus en plus présent et offrent de meilleurs performances que l'actuel 2G. L'accs multiple par division de code et séquence directe (DS-CDMA) est une solution qui doit être implémenter dans de tels systèmes de communication mais, à cause de la limitation du spectre radio et de l'émergence de nouvelles applications de plus en plus demandeuses en débit, il semble que la congestion dans ce type de systèmes restera un problème. Appliquer un modèle de tarification semble une solution simple pour résoudre ce problème. L'idée de cet article est d'étudier l'impact d'un modèle de tarification à l'usage statique par paquet sur la voix montante dans une cellule d'un réseau sans fils. Nous considérons que la demande (définie àl'aide des fonctions d'utilité) décroit quand les prix augmentent ou quand la qualité de service diminue. Cet article traite également d'une tarification avec trafic hétérogène car la technologie DS-CDMA supporte l'intégration de services. Tout d'abord, nous déterminons par un équilibre de Nash le nombre d'utilisateurs rejoignant le système en fonction de la demande. Ensuite, supposant un contrôle de puissance parfait, nous déterminons les prix et puissances de réception qui optimisent le revenu du fournisseur de service. Nous montrons que lorsque la demande excède toujours la capacité, le fournisseur maximise son revenu en servant une seul classe de service. Seulement, ceci n'est pas vraie dans le cas plus réaliste de demande aléatoire dans le temps.

Mots clés : Tarification, Réseaux sans-fils CDMA, Optimisation 


\section{Introduction}

With the widespread use of the Internet, telecommunications are nowadays of common use in our daily life. Moreover, the Internet is expected to converge into a single network with heterogeneous networks, like wireless and cable networks. Due to this convergence and new emerging applications such as multimedia, the network will deal with services with a large range of quality of service (QoS) requirements. This is combined with an increasing number of subscribers, each of them demanding a particular bandwidth. If the user demand is below network capacity, like in the Internet backbone, then congestion would not occur and there would be no special need for applying service differentiation since all QoS requirements would be likely satisfied. On the other hand, in access networks in general (where the problem is often called the last mile problem [2]), and in wireless communications in particular, capacity (the radio spectrum for wireless communications) will be hardly increased, and the growing demand needs to be managed in a way that "most" of demands are satisfactorily served. In this situation, it seems important to apply control and/or service differentiation procedures. In this paper, we focus on direct-sequence code-division multiple-access (DS-CDMA) networks that will form the next generation of wireless networks. DS-CDMA $([9,26])$ is indeed a way to control QoS, by appropriately selecting the transmission (meaning received) powers, which can be increased when the interference increases in order to satisfy the requested signal qualities. We consider the case of integrated services, that are supported in DS-CDMA, where multiple classes of service are provided (through different prices and received powers at the base station). We assume perfect power control at the base station, which is known to be crucial especially for the reverse-link [7, 21]: all signal powers of mobile users received at the base station are thus forced to be equal within a given class, avoiding near-far effects for instance.

Many papers have been devoted to power allocation and QoS management [18, 10, 11, 12, 13]. In these works, resource allocation schemes are proposed to provide the best possible QoS levels to clients, but they do not look at practical ways of controlling demand. To tackle out this problem, pricing is a simple and convenient approach. Pricing has been extensively studied in wired networks such as the Internet (see $[3,4,5,25]$ and the references therein) for controlling congestion and for differentiating services. It has also been used in CDMA networks ([1, 14, 16, 19, 20, 22, 23, 24]) by using their specificities: the price charged to a user is computed in terms of the QoS degradation the presence of this user imposes to others the so-called externality. This can be shown to directly depend on the transmission powers through the interferences. This generally leads to a game-theoretical analysis and price optimization.

We consider in this paper a different view of CDMA network control where prices do not depend on power or interference levels, but simply on the volume of transmitted data. Since in the scheme we propose prices do not integrate the real externality, it might be seen mathematically less efficient in terms of fairness or social welfware. However, we believe that such a volume-based pricing scheme will be more likely accepted by subscribers since it is more predictable. Also, we look for a static pricing scheme, where prices are fixed and do not vary with the network conditions. This is assumed for the same reasons, since again, we argue as in [17] that users would prefer to have an a-priori knowledge of the applied charge rather than a dynamic and random one, even if this is larger in average.

PI ${ }^{\circ} 1731$ 
We thus consider a pricing mechanism to optimize the network revenue in reverse-link DSCDMA transmissions. The model we propose is inspired by the one in [10], where an optimal resource allocation scheme was obtained among different classes of users, but for fixed and predetermined numbers of users in each class. In that paper, power is controlled to reach the given thresholds of signal-to-interference plus noise ratio (SINR) for which QoS requirements are met. A processing gain exhibiting good performance is computed. We consider here that the processing gain is fixed for each class of service, but on the other hand we compute the received powers allowing to optimize the network revenue. Our goal, with respect to [10], is to study how pricing can be used to control the number of users in the network and how, by means of pricing and received powers, the provider's revenue can be optimized. The introduction of demand with respect to prices and perceived QoS levels is obtained by introducing the so-called utility functions. These functions depend on both the QoS parameters and prices. QoS parameters vary with the type of traffic considered, for instance data is sensitive to delay, while voice is rather sensitive to losses and throughput, if delay is bounded. The better the quality, the more users will access the network, but the higher the prices, the less users will likely enter the network. We thus look at this trade-off as well as the trade with received power. With respect to [10], for dynamic range limitation on the multi-access receiver, we also introduce a capacity constraint representing the fact that only a finite number of customers can be received at the base station, this number depends on the reception power level [26]. The pricing problem is investigated when one or two different classes of traffic are involved. We consider situations where demand always exceeds capacity, but also cases where demand is random. The random case catches, for instance, the demand behavior over a full day since demand could be under capacity at some point of time. At a given time, we look for an equilibrium situation where demand adapts itself to prices and to received power requirements. Then, we look at prices and powers optimizing the provider's revenue.

This paper is organized as follows. In Section 2, we describe the basic model taken from [10], and then describe how demand varies with QoS and prices by using utility functions. Section 3 describes the case of a single class of users and Section 4 does the same analysis but in the case of two classes. Special attention is devoted to the equilibrium situation, especially for two classes, where users of both classes of traffic compete for resources. We consider the case where demand exceeds capacity, but also the case where potential demand may be under capacity. Finally, we conclude and give our directions for future research in Section 5. Proofs are left to appendices to ease the understandig of results.

\section{Model}

\subsection{CDMA Model}

The model we propose is based on the one in [10]. We focus on the reverse link of a single cell. We consider a DS-CDMA network (see $[9,26]$ for details), where the chip rate $R_{c}$ is assumed equal for all users. We assume that we have a multiclass system, with $C$ classes, where a user is characterized by a class $i$. When packets are sent, they enter a buffer after error control coding through forward error correction (FEC), and are converted to a DS-CDMA signal at symbol rate $R_{c} / N_{i}$, with $N_{i}$ 
being the processing gain (which should not be larger than $R_{c} /\left(\lambda_{i} L_{i}\right) . L_{i}$ is the length in terms of symbols of packet of class $i$. The signal transmission power is controlled such that it is received at level $P_{i}$ at the base station. The choice of $N_{i}$ and received power $P_{i}$ at the base station affects packet delay and transmission rate. This has been extensively discussed in [10]. Note that this also affects the performance of other classes of users. So, we fix the values of $N_{i}$ to the ones giving good performance in [10]. We consider that a new packet is generated as soon as the preceding one is successfully delivered. This is referred as continuously active users, which might represent the transmission of long files for instance.

In DS-CDMA, a key parameter is the received signal-to-interference plus noise-ratio (SINR). QoS metrics such as delay and bit error probability depend directly on it. For class $i$ users, the SINR is

$$
S I N R_{i}=\frac{P_{i} N_{i}}{\gamma\left(\sum_{k=1}^{K_{i}-1} P_{i}+\sum_{j \neq i}^{C} \sum_{k=1}^{K_{j}} P_{j}\right)+\sigma^{2}},
$$

where $\gamma$ is a constant which depends on the shape of DS-CDMA chips, $K_{j}$ is the number of class $j$ connections and $\sigma^{2}$ is the background noise power.

For all classes of traffic, we assume that channel coding includes forward error correction (FEC). We assume that the bit error probability (BEP) is an exponentially decaying function of the SINR. Specifically, we assume that for a user in class $i$, the BEP is

$$
p_{b_{i}}=\mathcal{F}\left(S I N R_{i}\right),
$$

with $\mathcal{F}(x)=\exp (-\beta x)^{1}$. Similarly, the probability of retransmission is

$$
p_{r_{i}}=1-\left[1-\mathcal{F}\left(S I N R_{i}\right)\right]^{L_{i} r_{i}}
$$

with $r_{i}$ the FEC code rate for class $i$.

Performances measures can be directly expressed in terms of the SINR. Consider for instance the mean packet delay $\mathbb{E} D_{i}$ for type-i traffic. It is composed of the mean waiting time in the queue $\mathbb{E} W_{i}$ and the mean retransmission time $\mathbb{E} S_{i}, \mathbb{E} D_{i}=\mathbb{E} W_{i}+\mathbb{E} S_{i}$. It is shown in [10] that

$$
\mathbb{E} D_{i}=\frac{L_{i} N_{i}}{R_{c}\left(1-p_{r_{i}}\right)} \text {. }
$$

On the other hand, base stations also have constraints on capacity. As stated in [26], for dynamic range limitations on the multiaccess receiver and to guarantee system stability, the total received noise plus interference power to background noise ratio is limited for a class- $i$ user to

$$
\frac{\gamma\left(\sum_{k=1}^{K_{i}-1} P_{i}+\sum_{j \neq i}^{C} \sum_{k=1}^{K_{j}} P_{j}\right)+\sigma^{2}}{\sigma^{2}} \leq \frac{1}{\eta}
$$

where $\eta$ is typically 0.25 or 0.1 . This inequality provides an upper-bound on the number of users for each class, for fixed received powers.

\footnotetext{
${ }^{1}$ In [10], a function $\mathcal{F}(x)=\kappa \mathrm{ep}(-\beta x)$ is rather used, implying that the delay of transmission is bounded even if the power is reduced to zero. To prevent this degenerated case, we adopt the approach used in [6], where $\kappa=1$ so that the delay of transmission is infinite and the probability of retransmission is equal to one when the power is zero.
} 


\subsection{Modelling users' behavior}

Let the class index $i$ be in $\{v, d\}$, where $v$ could be for voice traffic and $d$ for data. We abusively use this notation to keep in mind that class- $v$ is more sensitive to some QoS metrics (like delay) than class- $d$. The index is simply skipped when only one class is considered.

In general, a utility function $U_{i}$ is associated with a user of class $i(i \in\{v, d\})$, describing his level of satisfaction when transmitting a packet. This utility function is expressed as the difference between the value of the QoS level (depending on the SINR, which is function of the number of users of each type $K_{v}$ and $K_{d}$ ) and the per-packet charge $u_{i}$ for class $i$ :

$$
U_{i}\left(K_{v}, K_{d}\right)=f_{i}\left(\operatorname{SINR} R_{i}\left(K_{v}, K_{d}\right)\right)-u_{i} .
$$

$f_{i}$ describes how the valuation for service evolves with the SINR.

Assumption 1 We assume that the valuation function $f_{i}$ is strictly increasing, differentiable and that is such that $f_{i}(0)=0$ for all $i \in\{d, v\}$.

We will specifically assume that the utility function for class- $i$ traffic $(i \in\{d, v\})$ depends on the mean delay by

$$
U_{i}\left(K_{v}, K_{d}\right)=\frac{1}{\left(\mathbb{E} D_{i}\left(K_{v}, K_{d}\right)\right)^{\alpha_{i}}}-u_{i},
$$

where $\alpha_{i}$ is the sensitivity parameter of class- $i$ traffic to the mean delay (as considered in [15]) and $\mathbb{E} D_{i}\left(K_{v}, K_{d}\right)$ is given by (4). Note that $\mathbb{E} D_{i}\left(K_{v}, K_{d}\right)$ is a function of $\operatorname{SIN} R_{i}\left(K_{k}, K_{d}\right)$ fitting the above framework.

We assume, at least in a first step, that the number of potential sources is very large so that demand exceeds capacity. Selfish class $i$ users apply for service as soon as their utility $U_{i}$ is positive. Demand is thus directly controlled by prices and reception powers, so that it potentially leads to a (Nash) equilibrium on the number of active users where, for each class, either the number of sources is zero with negative utility (meaning that no user has interest in participating), or is equal to capacity with positive utility (meaning that no more users are allowed to enter for physical reasons), or the number of sources is positive and less than capacity, with null utility (meaning that the users' cost reach their valuation and no other user has interest in entering, since it would lead to a negative utility). Formally, an equilibrium is a tuple $\left(K_{v}^{*}, K_{d}^{*}\right)$ such that $K_{v}^{*}, K_{d}^{*} \geq 0$ and $\forall i, j \in\{d, v\}$, $j \neq i$ :

- Either $K_{i}^{*}=0$ and $U_{i}\left(1, K_{j}^{*}\right)<0$;

- or $K_{i}^{*}$ has reached the capacity constraint (5) (so the inequality becomes an equality) and $U_{i}\left(K_{v}^{*}, K_{d}^{*}\right)>0$;

- or $K_{i}^{*}>0$, under the capacity contraint (5), and $U_{i}\left(K_{v}^{*}, K_{d}^{*}\right)=0$ so that no other user has an incentive to join (a potentially leaving user being immediately replaced by a new one).

This leads to two different problems that we try to solve in the following sections: 
- What is the steady-state number of sources for fixed prices and reception powers? Is there a (unique) equilibrium, especially when considering two different classes of users in competition?

- What are the prices and powers that the service provider at the base station should set in order to maximize his revenue?

\section{Optimal pricing for a single class of users}

We consider in this section that the system has a single class of users. We first analyze the case where demand always exceeds capacity, then the case where demand is random and may at some point be under capacity. Recall that index $v$ or $d$ is skipped in this section.

\subsection{Demand exceeding capacity}

We assume that demand exceeds capacity. So, there always are users wishing to apply for service when their residual utility is positive. We first consider the case of a general utility function and then the case of a typical function that depends on the mean packet delay.

\subsubsection{General utility function}

With full generality, assume that the utility function of a user is expressed by

$$
U(K)=f(S I N R(K))-u,
$$

where function $f$ is positive, continuous, differentiable, strictly increasing and is such that $f(0)=0$ (Assumption 1).

The following theorem gives the number of sources at equilibrium in terms of fixed price and power.

Theorem 1 Let $u$ be the per-packet price and $P$ be the received power at the base station for all users. The number of users $K^{*}$ at equilibrium is:

$$
K^{*}= \begin{cases}0, & \text { if } u>u_{\max }=f\left(\frac{N P}{\sigma^{2}}\right), \\ 1-\frac{\sigma^{2}}{\gamma P}-\frac{N}{\gamma f^{-1}(u)}, & \text { if } f\left(\frac{N P \eta}{\sigma^{2}}\right)<u<f\left(\frac{N P}{\sigma^{2}}\right), \\ 1+\frac{1-\eta}{\eta} \frac{\sigma^{2}}{\gamma P}, & \text { otherwise. }\end{cases}
$$

The first and third cases are border situations: the first case corresponds to the situation where access is too expensive for users, the third case to the situation where full power capacity is reached. The proof of this theorem can be found in Appendix A.

With the number of sources determined, the second step is to look for per-packet price and received power maximizing the revenue of the base station. This problem can be formalized as

$$
\max _{u>0, P>0} R(u, P)=u K^{*}(u, P) \mu(u, P),
$$


with $\mu(u, P)$ the average throughput for each user and $K^{*}(u, P)$ the equilibrium number of sources determined in the above theorem. The average throughput is the average number of bits successfully transmitted per second, i.e.

$$
\begin{aligned}
\mu(u, P) & =\frac{L r}{\mathbb{E} D(u, P)} \\
& =\frac{r R_{c}}{N}\left(1-\exp \left(-\beta \frac{P N}{\gamma\left(K^{*}(u, P)-1\right) P+\sigma^{2}}\right)\right)^{L r} .
\end{aligned}
$$

The following theorem gives the price and received power optimizing the base station revenue.

Theorem 2 Let $\mathcal{X}$ be the set of solutions of the following equation in $X>0$ :

$$
\begin{gathered}
f(X)\left(\gamma X^{2} \operatorname{Lr} \beta e^{-\beta X}+X N(1-\eta) \operatorname{Lr} \beta-N\left(1-e^{-\beta X}\right)\right) \\
+f^{\prime}(X)\left(\gamma X^{2}\left(1-e^{-\beta X}\right)+N X\left(1-e^{-\beta X}\right)\right)=0 .
\end{gathered}
$$

Let

$$
X^{*}=\arg \max _{X \in \mathcal{X}} \frac{r R_{c}}{N}\left(1-e^{-\beta X}\right)^{L r} f(X)+\frac{1-\eta}{\gamma} r R_{c} \frac{\left(1-e^{-\beta X}\right)^{L r} f(X)}{X} .
$$

Then the per-packet price $u^{*}$ and received power $P_{d}^{*}$ that maximise the base station revenue are

$$
u^{*}=f\left(X^{*}\right) \text { and } P^{*}=\frac{X^{*} \sigma^{2}}{N \eta} .
$$

The proof is in Appendix B.

\subsubsection{Utility function depending on the average delay}

Assume now more specifically that the valuation of users depends on the average delay as $\frac{1}{(\mathbb{E} D)^{\alpha}}$ (as used in [8]). So, the utility is $U(K)=\frac{1}{\mathbb{E} D^{\alpha}}-u$. The function $f$ such that $f(S I N R(K))=\frac{1}{(\mathbb{E} D)^{\alpha}}$ is

$$
f(x)=\left(\frac{R_{c}}{L N}\right)^{\alpha}\left(1-e^{-\beta x}\right)^{L r \alpha}
$$

from (3) and (4). Note that this function is positive, continuous, differentiable, strictly increasing and is such that $f(0)=0$. Theorems 1 and 2 can be be restated as follows.

Corollary 1 Under the assumption that the utility function depends on the average packet delay:

- the number of users $K^{*}$ at equilibrium as a function of $(P, u)$ is

$$
K^{*}= \begin{cases}0, & \text { if } u>u_{\max }, w i t h u_{\max }=\left(\frac{R_{c}}{L N}\right)^{\alpha}\left(1-e^{-\beta \frac{N P}{\sigma^{2}}}\right)^{\operatorname{Lr} \alpha} . \\ 1-\frac{\sigma^{2}}{\gamma P}-\frac{\beta N}{\gamma \ln \left(1-\left(\frac{L N}{R_{c}} u^{1 / \alpha}\right)^{1 / L r}\right)}, & \text { if }\left(\frac{R_{c}}{L N}\right)^{\alpha}\left(1-e^{-\beta \frac{N P \eta}{\sigma^{2}}}\right)^{L r \alpha}<u<\left(\frac{R_{c}}{L N}\right)^{\alpha}\left(1-e^{-\beta \frac{N P}{\sigma^{2}}}\right)^{L r \alpha} . \\ 1+\frac{1-\eta}{\eta} \frac{\sigma^{2}}{\gamma P}, & \text { oheris } .\end{cases}
$$


- Let $X^{*}$ be the unique positive solution of the equation

$$
\operatorname{Lr}(1+\alpha) \beta X^{2}+\frac{1-\eta}{\gamma} \operatorname{Lr}(1+\alpha) \beta N X+(1-\eta) \frac{N}{\gamma}=\frac{1-\eta}{\gamma} N e^{\beta X}
$$

The optimal per-packet price is:

$$
u^{*}=\left(\frac{R_{c}}{L N}\right)^{\alpha}\left(1-e^{-\beta X^{*}}\right)^{\alpha L r},
$$

and the optimal received power is:

$$
P^{*}=\frac{X^{*} \sigma^{2}}{N \eta}
$$

This result is proved in Appendix C.

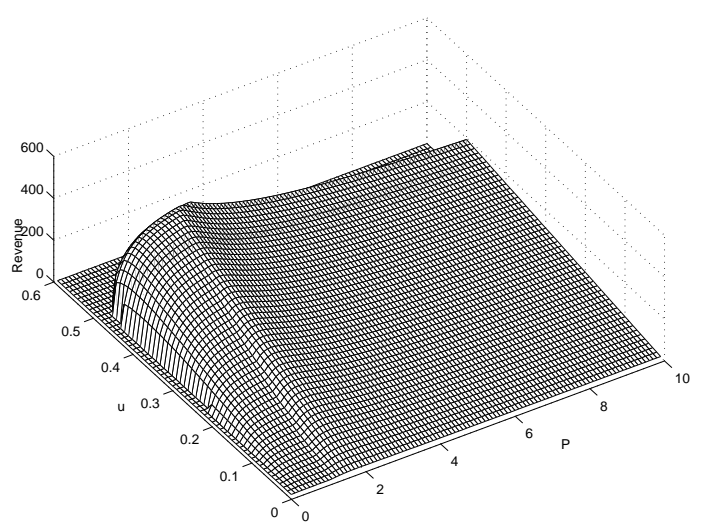

Figure 1: Revenue of the base station in terms of the per-packet price and the received power when demand exceeds capacity and delay is the metrics of interest.

As a numerical example, we consider the following variables: $L=768$ symbols, $r=1 / 2$, $\beta=2, \gamma=2 / 3$ for rectangular chips, $R_{c}=5$ Mchips $/ \mathrm{s}, \sigma^{2}=0.9 \mathrm{~dB}, \alpha=1.6$ and $N=10$. We also consider the upper bound of the power ratio $\eta=0.2$. Corollary 1 gives the optimal perpacket price $u^{*}=0.4804$ and the optimal received power $P^{*}=2.1352$. The maximal revenue is $R^{*}=411.699$. The revenue is displayed in Figure 1 in terms of the per-packet price $u$ and the received power $P$; it can be observed that this is in agreement with the optimization results.

\subsection{Random demand}

We assume in this subsection that demand varies so that, during a portion of time, it does not exceed capacity. Assume that demand is expressed by a discrete random variable $\Delta$ representing the 
number of potential users requesting service. The overall goal is again to determine the fixed price $u$ and power $P$ (remember that we look for a static pricing) that maximize the expected base station revenue. To reach this goal, we first need to look at the number of users for each possible level of demand (exceeding capacity or not), whatever the choice of $u$ and $P$.

Theorem 3 Let $u$ and $P$ be the per-packet price and received power at the base station. Let $\delta$ be the number of users potentially requesting service. The actual number of number of users $\kappa^{*}$ at equilibrium is

$$
\kappa^{*}=\min \left(\delta, K^{*}\right),
$$

with $K^{*}$ being the value found in Theorem 1 when demand exceeds capacity.

The proof of this theorem is in Appendix D.

The average base station revenue is expressed by:

$$
\bar{R}(u, P)=\sum_{\delta=0}^{\infty} u \kappa^{*}(u, P, \delta) \mu(u, P, \delta) \mathbb{P}(\Delta=\delta)
$$

where $\kappa^{*}(u, P, \delta)$ (resp. $\mu(u, P, \delta)$ ) is the number of open connections (resp. the throughput) when per-packet price is $u$, received power is $P$ and potential demand is $\delta$.

The goal of the base station is, again, to find a price $u$ and a power $P$ that maximize the expected revenue, representing this revenue over long periods of time. For instance, assume that demand $\Delta$ follows a Poisson distribution with rate 3, the other parameters being the same than for the example when demand exceeds capacity. By using standard optimization tools we get the optimal values $u^{*}=0.4905, P^{*}=2.2694$ and the maximum average revenue $\bar{R}^{*}=299.2589$. This is sketched in Figure 2.

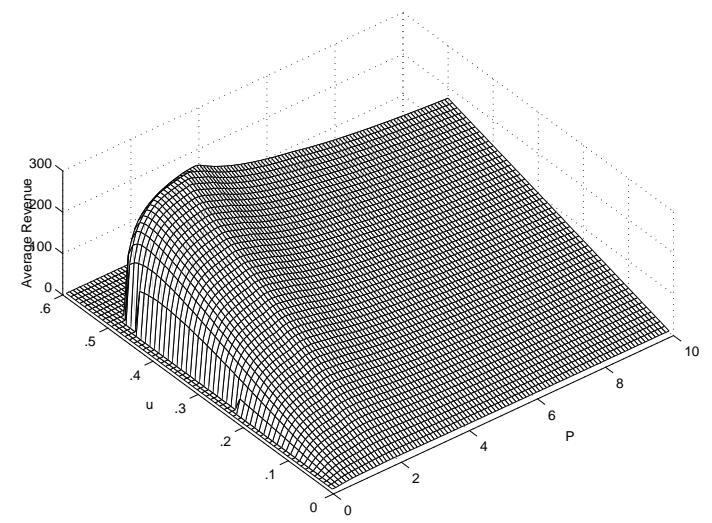

Figure 2: Average revenue of the base station in terms of the per-packet price and the received power when demand is random and delay is the metrics of interest. 


\section{Optimal pricing for two classes of users}

Consider now two different classes of applications with different quality of service valuation for which we want to differentiate services. For convenience, these classes are called type- $v$ and type- $d$. Here again, we assume that the service valuation for each type of application depends of the $S I N R$ obtained. This is given by $f_{v}\left(S I N R_{v}\left(K_{v}, K_{d}\right)\right)$ (resp. $f_{d}\left(S I N R_{d}\left(K_{v}, K_{d}\right)\right)$ ) for type- $v$ (resp. type- $d$ ) users. Therefore, if $u_{v}$ (resp. $u_{d}$ ) is the per-packet price for type- $v$ (resp. type- $d$ ), the utility functions for each type are

$$
\forall i \in\{v, d\}, \quad U_{i}\left(K_{v}, K_{d}\right)=f_{i}\left(\operatorname{SINR}_{i}\left(K_{v}, K_{d}\right)\right)-u_{i} .
$$

The $S I N R$ s, obtained from (1), are given by

$$
\begin{aligned}
\operatorname{SINR}_{v}\left(K_{v}, K_{d}\right) & =\frac{P_{v} N_{v}}{\gamma\left(K_{v}-1\right) P_{v}+\gamma K_{d} P_{d}+\sigma^{2}} \\
\operatorname{SINR}_{d}\left(K_{v}, K_{d}\right) & =\frac{P_{d} N_{d}}{\gamma K_{v} P_{v}+\gamma\left(K_{d}-1\right) P_{d}+\sigma^{2}}
\end{aligned}
$$

and, as it can be readily checked, the utility function of a type of users depends on the number of active users of the other type.

Again, we assume that there is a capacity constraint at the base station for each class, given by (5). These requirements lead to

$$
\begin{gathered}
K_{v}<\frac{1-\eta}{\eta} \frac{\sigma^{2}}{\gamma P_{v}}-K_{d} \frac{P_{d}}{P_{v}}+1(\text { for class }-v), \\
K_{v}<\frac{1-\eta}{\eta} \frac{\sigma^{2}}{\gamma P_{v}}-K_{d} \frac{P_{d}}{P_{v}}+\frac{P_{d}}{P_{v}}(\text { for class- } d),
\end{gathered}
$$

or just

$$
K_{v}<\frac{1-\eta}{\eta} \frac{\sigma^{2}}{\gamma P_{v}}-K_{d} \frac{P_{d}}{P_{v}}+1+\frac{P_{d}-P_{v}}{P_{v}} \mathbb{1}_{P_{d}<P_{v}}
$$

where 11 is for the indicator function.

\subsection{Demand exceeding capacity}

We again first assume that the number of users potentially applying for service is infinite in each type of application. The case of random demand is studied in the next subsection.

We assume users act selfishly and apply for service as soon as their utility is positive. Also, they leave the game if their utility becomes negative. Again, as in the single class case, the base station blocks connections from users if the capacity constraint (12) is reached.

The following theorem shows that a Nash equilibrium exists for the number of users of each type, when considering that all users are selfish. The theorem expresses this equilibrium in terms of prices and received powers for each class of service.

PI ${ }^{\circ} 1731$ 
Theorem 4 With the above assumptions, there is a (Nash) equilibrium $\left(K_{v}^{*}, K_{d}^{*}\right)$ for the number $K_{v}^{*}$ and $K_{d}^{*}$ of active type-v and type-d users. The values of $K_{v}^{*}$ and $K_{d}^{*}$ depend on received powers $P_{v}, P_{d}$ and prices $u_{v}, u_{d}$ in the following fashion:

1. If $U_{v}(1,0) \leq 0 \Leftrightarrow u_{v} \geq f_{v}\left(\frac{N_{v} P_{v}}{\sigma^{2}}\right)$ and $U_{d}(0,1) \leq 0 \Leftrightarrow u_{d} \geq f_{d}\left(\frac{N_{d} P_{d}}{\sigma^{2}}\right)$, no user is interested in requesting service, so that

$$
\left(K_{v}^{*}, K_{d}^{*}\right)=(0,0) .
$$

2. If $U_{v}(1,0) \leq 0 \Leftrightarrow u_{v} \geq f_{v}\left(\frac{N_{v} P_{v}}{\sigma^{2}}\right)$ but $U_{d}(0,1)>0$, only type-d users are present at equilibrium and the Nash equilibrium is $\left(0, K_{d}^{*}\right)$ with

$$
K_{d}^{*}= \begin{cases}1-\frac{\sigma^{2}}{\gamma P_{d}}-\frac{N_{d}}{\gamma f_{d}^{-1}\left(u_{d}\right)}, & \text { if } f_{d}\left(\frac{N_{d} P_{d} \eta}{\sigma^{2}}\right)<u_{d}<f_{d}\left(\frac{N_{d} P_{d}}{\sigma^{2}}\right), \\ 1+\frac{1-\eta}{\eta} \frac{\sigma^{2}}{\gamma P_{d}}, & \text { otherwise. }\end{cases}
$$

3. On the other hand, if $U_{d}(0,1) \leq 0 \Leftrightarrow u_{d} \geq f_{d}\left(\frac{N_{d} P_{d}}{\sigma^{2}}\right)$ but $U_{v}(1,0)>0$, only type-v users are present and the Nash equilibrium is $\left(K_{v}^{*}, 0\right)$ with

$$
K_{v}^{*}= \begin{cases}1-\frac{\sigma^{2}}{\gamma P_{v}}-\frac{N_{v}}{\gamma f_{v}^{-1}\left(u_{v}\right)}, & \text { if } f_{v}\left(\frac{N_{v} P_{v} \eta}{\sigma^{2}}\right)<u_{v}<f_{v}\left(\frac{N_{v} P_{v}}{\sigma^{2}}\right), \\ 1+\frac{1-\eta}{\eta} \frac{\sigma^{2}}{\gamma P_{v}}, & \text { otherwise. }\end{cases}
$$

4. If $U_{v}(1,0)>0$ and $U_{d}(0,1)>0$, there are 3 subcases.

(a) If $\frac{\sigma^{2}}{\eta \gamma}+P_{v}+\left(P_{d}-P_{v}\right) \mathbb{1}_{P_{d}<P_{v}} \leq \min \left(\frac{N_{d} P_{d}}{\gamma f_{d}^{-1}\left(u_{d}\right)}+P_{d}, \frac{N_{v} P_{v}}{\gamma f_{v}^{-1}\left(u_{v}\right)}+P_{v}\right)$, the capacity constraint (12) is reached while users of both classes still want to request service. The system then chooses to accept only class- $d$ users if $u_{d}>\frac{P_{d} \mu_{v}^{c}}{P_{v} \mu_{d}^{c}} u_{v}$, or only class- $v$ users otherwise in order to give priority to users providing the highest revenue, with $\forall i \in$ $\{v, d\}$ the throughput at capacity

$$
\mu_{i}^{c}=\frac{r R_{c}}{N_{i}}\left(1-e^{-\beta \frac{N_{i} P_{i}}{\sigma^{2} / \eta+\gamma\left(P_{j}-P_{i}\right) 1_{P_{j}<P_{i}}}}\right)^{L r} .
$$

The equilibrium is thus $\left(0, K_{d}^{*}\right)$ with $K_{d}^{*}=1+\frac{1-\eta}{\eta} \frac{\sigma^{2}}{\gamma P_{d}}$ if $u_{d}>\frac{P_{d} \mu_{v}^{c}}{P_{v} \mu_{d}^{c}} u_{v}$, and $\left(K_{v}^{*}, 0\right)$ with $K_{v}^{*}=1+\frac{1-\eta}{\eta} \frac{\sigma^{2}}{\gamma P_{v}}$ otherwise.

(b) If $\frac{N_{d} P_{d}}{\gamma f_{d}^{-1}\left(u_{d}\right)}+P_{d}<\min \left(\frac{\sigma^{2}}{\eta \gamma}+P_{v}+\left(P_{d}-P_{v}\right) \mathbb{1}_{P_{d}<P_{v}}, \frac{N_{v} P_{v}}{\gamma f_{v}^{-1}\left(u_{v}\right)}+P_{v}\right)$, the equilibrium is $\left(K_{v}^{*}, 0\right)$ with $K_{v}^{*}$ defined in (14).

(c) Else if $\frac{N_{v} P_{v}}{\gamma f_{v}^{-1}\left(u_{v}\right)}+P_{v}<\min \left(\frac{N_{d} P_{d}}{\gamma f_{d}^{-1}\left(u_{d}\right)}+P_{d}, \frac{\sigma^{2}}{\eta \gamma}+P_{v}+\left(P_{d}-P_{v}\right) \mathbb{1}_{P_{d}<P_{v}}\right)$, the equilibrium is $\left(0, K_{d}^{*}\right)$ with $K_{d}^{*}$ defined in (13). 
This theorem is proved in Appendix E.

It can be observed that, at equilibrium, only one type of application is in service when demand exceeds capacity.

Knowing this equilibrium, one can obtain the values of prices $u_{v}^{*}, u_{d}^{*}$ and the received power $P_{v}^{*}, P_{d}^{*}$ that maximizes the network revenue.

Theorem 5 The maximum revenue of the base station is

$$
R^{*}=\max \left(R_{v}^{*}, R_{d}^{*}\right)
$$

where $R_{v}^{*}$ (resp. $R_{d}^{*}$ ) is the maximum revenue when there are only class- $v$ (resp. class-d) users, provided by Theorem 2 (single-class case).

Let $i \in\{v, d\}$ such that $R_{i}^{*}=R^{*}$ and $j \in\{v, d\}$ such that $j \neq i$. The class- $i$ optimal perpacket price is $u_{i}^{*}=f_{i}\left(X^{*}\right)$ and the optimal received power $P_{i}^{*}=\frac{X^{*} \sigma^{2}}{N_{i} \eta}$ where class $-i$ is used in Theorem 2 to obtain $X^{*}$.

Values $u_{j}^{*}$ and $P_{j}^{*}$ for the other class must be chosen so that only class- $i$ users are present at equilibrium, that is, they should verify $\forall P_{j}^{*}>0$

$$
u_{j}^{*} \in\left[0, \min \left(\frac{P_{j}^{*} \mu_{i}^{c}}{P_{i}^{*} \mu_{j}^{c}} u_{i}^{*}, f_{j}\left(\frac{P_{j}^{*} N_{j}}{\sigma^{2}} \eta\right)\right)\left[\cup \left[f_{j}\left(\frac{P_{j}^{*} N_{j}}{\sigma^{2}} \eta\right),+\infty[.\right.\right.\right.
$$

The proof is provided in Appendix F.

As an illustration, consider again the case where the valuation depends on the average delay for each class. We use the same numerical values than in the case of a single class, those values corresponding to type- $d$ users. We additionally use the following processing gain $N_{v}=8$ and the delay sensitivity $\alpha_{v}=2$, so that voice users value more small delays. It turns out that, at equilibrium, only type- $v$ users are present, providing a revenue $R^{*}=574.063$, obtained for values $u_{v}^{*}=0.63$ and $P_{v}^{*}=2.74$.

The revenue is thus maximized when there is only one class of user in the system at equilibrium. This result is based on the strong assumption that potential demand always exceeds capacity. Though, it is likely that at some point of time, demand is less than capacity. This occurs for instance during the evening. In the next section, we assume that demand follows a random variable, and we keep the idea of having static (fixed) prices (as well as received powers). Therefore, keeping two classes of service is useful, both classes being served, but the most important one getting all resource in case of congestion.

\subsection{Random Demand}

We assume that $\forall i \in\{v, d\}, \Delta_{i}$ defines the (discrete) random demand for type- $i$ users. Given the values $\delta_{i}$ of random variables $\Delta_{i}$, an equilibrium exists for the numbers $\kappa_{v}^{*}$ and $\kappa_{d}^{*}$ of type- $v$ and type- $d$ users.

Theorem 6 Assuming now that potential demand is $\delta_{v}$ and $\delta_{d}$ for type- $v$ and type-d users respectively and that it does not necessarily exceed capacity, there is a (Nash) equilibrium $\left(\kappa_{v}^{*}, \kappa_{d}^{*}\right)$ for the 
numbers $\kappa_{v}^{*}$ and $\kappa_{d}^{*}$ of active type-v and type-d users. The values of $\kappa_{v}^{*}$ and $\kappa_{d}^{*}$ depend on received powers $P_{v}, P_{d}$, prices $u_{v}, u_{d}$, and potential demand $\delta_{v}, \delta_{d}$ in the following way:

1. if ( $\delta_{v}=0$ or $U_{v}(1,0) \leq 0 \Leftrightarrow u_{v} \geq f_{v}\left(\frac{N_{v} P_{v}}{\sigma^{2}}\right)$ ) and $\left(\delta_{d}=0\right.$ or $U_{d}(0,1) \leq 0 \Leftrightarrow u_{d} \geq$ $\left.f_{d}\left(\frac{N_{d} P_{d}}{\sigma^{2}}\right)\right)$, no user has interest in requesting service, so that

$$
\left(\kappa_{v}^{*}, \kappa_{d}^{*}\right)=(0,0)
$$

2. If $\delta_{v}=0$ or $U_{v}(1,0) \leq 0 \Leftrightarrow u_{v} \geq f_{v}\left(\frac{N_{v} P_{v}}{\sigma^{2}}\right)$ but $\delta_{d}>0$ and $U_{d}(0,1)>0$, only type-d users are present at equilibrium and the Nash equilibrium is $\left(0, \kappa_{d}^{*}\right)$ with

$$
\kappa_{d}^{*}=\min \left(\delta_{d}, K_{d}^{*}\right),
$$

$K_{d}^{*}$ being taken from (13).

3. On the other hand, if $\delta_{d}=0$ or $U_{d}(0,1) \leq 0 \Leftrightarrow u_{d} \geq f_{d}\left(\frac{N_{d} P_{d}}{\sigma^{2}}\right)$ but $\delta_{v}>0$ and $U_{v}(1,0)>$ 0 , only type-v users are present and the Nash equilibrium is $\left(\kappa_{v}^{*}, 0\right)$ with

$$
\kappa_{v}^{*}=\min \left(\delta_{v}, K_{v}^{*}\right)
$$

$K_{v}^{*}$ being taken from (14).

4. If the total demand is less than what capacity can support and it still yields positive utilities, that is if $\delta_{v}<\frac{1-\eta}{\eta} \frac{\sigma^{2}}{\gamma P_{v}}-\delta_{d} \frac{P_{d}}{P_{v}}+1+\frac{P_{d}-P_{v}}{P_{v}} \mathbb{1}_{P_{d}<P_{v}}, U_{v}\left(\delta_{v}, \delta_{d}\right)>0$ and $U_{d}\left(\delta_{v}, \delta_{d}\right)>0$, all users are served, i.e., $\left(\kappa_{v}^{*}, \kappa_{d}^{*}\right)=\left(\delta_{v}, \delta_{d}\right)$.

5. Otherwise, if $\delta_{v}, \delta_{d}>0, U_{v}(1,0)>0$, and $U_{d}(0,1)>0$, there are 3 subcases.

(a) If $\frac{\sigma^{2}}{\eta \gamma}+P_{v}+\left(P_{d}-P_{v}\right) \mathbb{1}_{P_{d}<P_{v}} \leq \min \left(\frac{N_{d} P_{d}}{\gamma f_{d}^{-1}\left(u_{d}\right)}+P_{d}, \frac{N_{v} P_{v}}{\gamma f_{v}^{-1}\left(u_{v}\right)}+P_{v}\right)$, capacity constraint (12) (and potential demand constraints $\left(\delta_{v}, \delta_{d}\right)$ ) are reached while users of both classes still have interest to request for service. The system then decides to give preference to class-d users if $u_{d}>\frac{P_{d} \mu_{v}^{c}}{P_{v}^{c} \mu_{d}^{c}} u_{v}$ or to class- $v$ users otherwise in order to prioritize users that provide a higher revenue.

The equilibrium is thus $\left(\kappa_{v}^{*}, \kappa_{d}^{*}\right)$ with

- $\kappa_{d}^{*}=\min \left(\delta_{d}, 1+\frac{1-\eta}{\eta} \frac{\sigma^{2}}{\gamma P_{d}}\right)$ and $\kappa_{v}^{*}=\frac{\frac{1-\eta}{\eta} \frac{\sigma^{2}}{\gamma}+P_{v}+\left(P_{d}-P_{v}\right) \mathbb{1}_{P_{d}<P_{v}}-\kappa_{d}^{*} P_{d}}{P_{v}}$ if $u_{d}>$ $\frac{P_{d} \mu_{v}^{c}}{P_{v} \mu_{d}^{c}} u_{v}$

- $\kappa_{v}^{*}=\min \left(\delta_{v}, 1+\frac{1-\eta}{\eta} \frac{\sigma^{2}}{\gamma P_{v}}\right)$ and $\kappa_{d}^{*}=\frac{\frac{1-\eta}{\eta} \frac{\sigma^{2}}{\gamma}+P_{v}+\left(P_{d}-P_{v}\right) \mathbb{1}_{P_{d}<P_{v}-\kappa_{v}^{*} P_{v}}}{P_{d}}$ otherwise.

(b) If $\frac{N_{d} P_{d}}{\gamma f_{d}^{-1}\left(u_{d}\right)}+P_{d}<\min \left(\frac{\sigma^{2}}{\eta \gamma}+P_{v}+\left(P_{d}-P_{v}\right) \mathbb{1}_{P_{d}<P_{v}}, \frac{N_{v} P_{v}}{\gamma f_{v}^{-1}\left(u_{v}\right)}+P_{v}\right)$, the equilibrium is $\left(\kappa_{v}^{*}, 0\right)$ with $\kappa_{v}^{*}$ defined in (16).

(c) Else if $\frac{N_{v} P_{v}}{\gamma f_{v}^{-1}\left(u_{v}\right)}+P_{v}<\min \left(\frac{N_{d} P_{d}}{\gamma f_{d}^{-1}\left(u_{d}\right)}+P_{d}, \frac{\sigma^{2}}{\eta \gamma}+P_{v}+\left(P_{d}-P_{v}\right) \mathbb{1}_{P_{d}<P_{v}}\right)$, the equilibrium is $\left(0, \kappa_{d}^{*}\right)$ with $\kappa_{d}^{*}$ defined in (15). 
The proof follows exactly that of Theorem 4 , just adding demand constraints $\delta_{v}$ and $\delta_{d}$ to capacity constraints.

In the current case of two classes, the average base station revenue is expressed by:

$$
\begin{aligned}
\bar{R}\left(u_{v}, P_{v}, u_{d}, P_{d}\right)= & \sum_{\delta_{v}=0}^{\infty} \sum_{\delta_{d}=0}^{\infty}\left(u_{v} \kappa_{v}^{*}\left(u_{v}, P_{v}, \delta_{v}, u_{d}, P_{d}, \delta_{d}\right) \mu_{v}\left(u_{v}, P_{v}, \delta_{v}, u_{d}, P_{d}, \delta_{d}\right)+\right. \\
& \left.+u_{d} \kappa_{d}^{*}\left(u_{v}, P_{v}, \delta_{v}, u_{d}, P_{d}, \delta_{d}\right) \mu_{d}\left(u_{v}, P_{v}, \delta_{v}, u_{d}, P_{d}, \delta_{d}\right)\right) \mathbb{P}\left(\Delta_{v}=\delta_{v}, \Delta_{d}=\delta_{d}\right),
\end{aligned}
$$

where $\forall i \in\{v, d\}, \kappa_{i}^{*}(u, P, \delta)$ (resp. $\mu_{i}(u, P, \delta)$ ) is the number of type- $i$ open connections (resp. the throughput) when per-packet prices are $u_{v}, u_{v}$, received powers $P_{v}, P_{d}$ and potential demand is $\delta_{v}, \delta_{d}$.

As an illustration, we look at the case where demand follows a Poisson distribution with rate 2 for type- $v$ traffic and 3 for type- $d$. We consider also the following parameters: $N_{d}=10, N_{v}=8$, $\alpha_{d}=1.6$ and $\alpha_{v}=2$. This choice gives the optimal values $u_{v}^{*}=0.638, u_{d}^{*}=0.484, P_{v}^{*}=2.635$, $P_{d}^{*}=2.177$ and maximum average revenue $R^{*}=504.836$. We plot in Figure 3 (resp. 4 ) the average revenue in terms of type- $d$ (resp. type- $v$ ) users received power and per-packet price with optimal type- $v$ (resp. type- $d$ ) parameters.

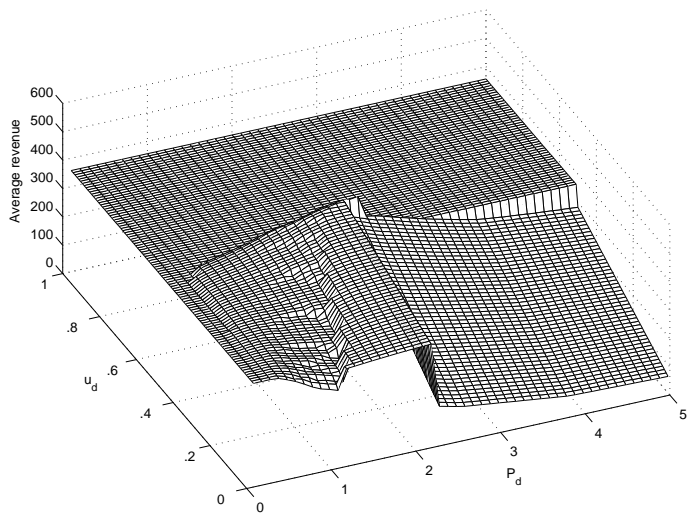

Figure 3: Average base station revenue in terms of type- $d$ users received power $P_{d}$ and per-packet price $u_{d}$ with optimal type- $v$ received power $P_{v}^{*}$ and per-packet price $u_{v}^{*}$.

The same thing is performed in Figures 5 and 6, but with their respective powers and prices varying. The values in the figures are in accordance with the optimal value found. 


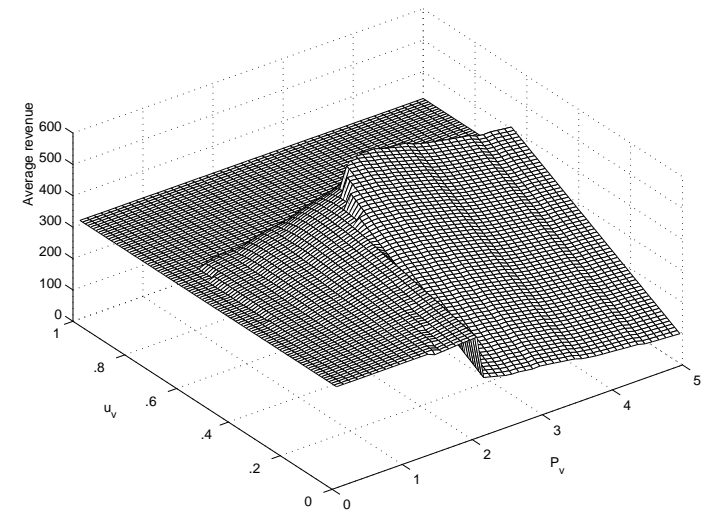

Figure 4: Average base station revenue in terms of type- $v$ users received power $P_{v}$ and per-packet price $u_{v}$ when type- $d$ parameters are fixed to $P_{d}^{*}$ and $u_{d}^{*}$.

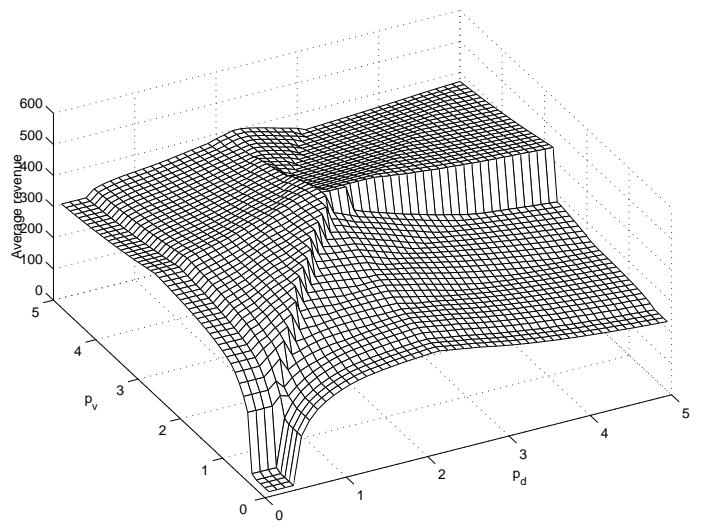

Figure 5: Average base station revenue in terms of received powers $P_{v}$ and $P_{d}$ with prices fixed to optimal values $u_{v}^{*}$ and $u_{d}^{*}$.

\section{Conclusions}

We have investigated in this paper a new pricing scheme for DS-CDMA communications, allowing service differentiation. With respect to the schemes developed in the literature, we have chosen a static and predictable per-packet price that, we believe, is more likely to be accepted by users. The base station (assuming perfect power control) controls two variables per class: the price and received power. For fixed values, we have found the number of users applying for service at equilibrium, 


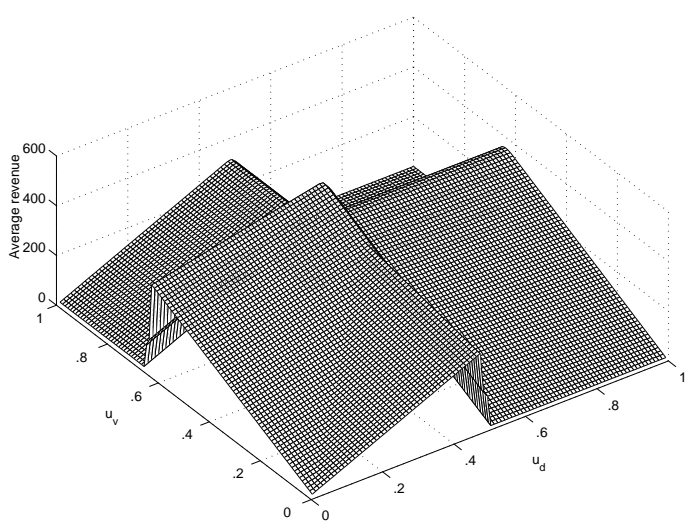

Figure 6: Average base station revenue in terms of per-packet prices $u_{v}$ and $u_{d}$ with received powers fixed to optimal values $P_{v}^{*}$ and $P_{d}^{*}$.

whatever demand is. We have also looked at the price and power values that maximize the revenue at the base station. Our findings show that, when demand exceeds capacity, one type of service will get the priority. On the other hand, assuming a more likely random demand, we have illustrated that both classes will be served.

As extensions of our results, we would like to look at the case where users are not continuously active, but their activity follow a random variable [10].

\section{References}

[1] T. Alpcan, T. Başar, R. Srikant, and E. Altman. CDMA uplink power control as a noncooperative game. Wireless Networks, 2002.

[2] L. Bernstein. Managing the last mile. IEEE Communications Magazine, 35(10):72-76, Oct 1997.

[3] C. Courcoubetis and R. Weber. Pricing Communication Networks-Economics, Technology and Modelling. Wiley, 2003.

[4] L.A. DaSilva. Pricing of QoS-Enabled Networks: A Survey. IEEE Communications Surveys \& Tutorials, 3(2), 2000.

[5] M. Falkner, M. Devetsikiotis, and I. Lambadaris. An Overview of Pricing Concepts for Broadband IP Networks. IEEE Communications Surveys \& Tutorials, 3(2), 2000.

PI ${ }^{\circ} 1731$ 
[6] D. Famolari, N. Mandayam, D. Goodman, and V. Shah. Wireless Multimedia Network Technologies, chapter A New Framework for Power Control in Wireless Data Networks: Games, Utility and Pricing, pages 289-310. Kluwer, 1999.

[7] S. Glisic and B. Vucetic. Spread Spectrum CDMA Systems for Wireless Communications. Artech House, 1997.

[8] Y. Hayel, D. Ros, and B. Tuffin. Less-than-best-effort services: Pricing and scheduling. In Proceedings of the IEEE Infocom, Hong Kong, March 2004.

[9] H. Holma and A. Toksala. WCDMA for UMTS, revised edition. John Wiley \& Sons Inc., 2001.

[10] J.B. Kim and M.L. Honig. Resource allocation for multiple classes of DS-CDMA traffic. IEEE Transactions on Vehicular Technology, 49(2):506-519, March 2000.

[11] J. Lee, R. Mazumdar, and N. Shroff. Downlink Power Allocation for Multi-class CDMA Wireless Networks. In Proceedings of INFOCOM, 2002.

[12] P. Liu, M.L. Honig, and S. Jordan. Forward-link CDMA resource allocation based on pricing. In Proceedings of the 2000 IEEE Wireless Communications and Networking Conference, pages 1410-1414, 2000.

[13] U. Madhow and M. L. Honig. Mmse interference suppression for direct-sequence spreadspectrum cdma. IEEE Transactions on Communications, 42(12):3178-3188, 1994.

[14] P. Maillé. Auctioning for downlink transmission power in CDMA cellular systems. In Proceedings of ACM/IEEE MSWiM, Oct 2004.

[15] M. Mandjes. Pricing strategies under heterogenous service requirements. In Proceedings of the IEEE Infocom, San Francisco, CA, USA, March 2003.

[16] P. Marbach and R. Berry. Downlink resource allocation and pricing for wireless networks. In Proceedings of IEEE Infocom, 2002.

[17] A. Odlyzko. The history of communications and its implications for the Internet. Technical report, AT\&T Labs, 2000.

[18] A. Sampath, N. Mandayam, and J.M. Holtzman. Analysis of an access control mechanism for data traffic in an integrated voice/data wireless cdma system. In Proceedings of the 46th Vehicular Technology Conference, 1996.

[19] C.U. Saraydar, N.B. Mandayam, and D.J. Goodman. Pricing and power control in a multicell wireless data network. IEEE JSAC Wireless Series, 19(2):277-286, 2001.

[20] C.U. Saraydar, N.B. Mandayam, and D.J. Goodman. Efficient power control via pricing in wireless data networks. IEEE transactions on Communications, 50(2):291-303, 2002. 
[21] S. Sigit Puspito, W. Jarot, and M. Nakagawa. Transmission power control techniques for the reverse link of OFDM-DS-CDMA system. In Proceedings of the Fourth IEEE Symposium on Computers and Communications, pages 331-337, 1999.

[22] V.A. Siris. Resource control for elastic traffic in cdma networks. In Proc. of MOBICOM'02, 2002.

[23] V.A. Siris. Resource control for elastic traffic in CDMA networks. In 8th international conference on Mobile computing and networking, pages 193-204, Atlanta, USA, 2002. ACM Press.

[24] V.A. Siris and C. Courcoubetis. Resource control for loss-sensitive traffic in cdma networks. In Proceedings of IEEE Infocom 2004, Hong-Kong, China, 2004.

[25] B. Tuffin. Charging the Internet without bandwidth reservation: an overview and bibliography of mathematical approaches. Journal of Information Science and Engineering, 19(5):765-786, 2003.

[26] A.J. Viterbi. CDMA. Principles of Spread Spectrum Communication. Addison-Wesley, 1995.

\section{A Proof of Theorem 1}

We consider that the per-packet price $u$ and received power $P$ are fixed. The goal is to find an equilibrium number of sources $K^{*}$ such that no additional users will have an incentive to join the system (and somehow no present users will want to leave). This means that we are looking for a value $K^{*}$ such that

- $U(1) \leq 0$ and $K^{*}=0$,

- or $U\left(K^{*}\right)>0$ and $K^{*}=\frac{1-\eta}{\eta} \frac{\sigma^{2}}{\gamma P}+1$ (this last equality corresponding to the case where capacity is reached in (5)),

- or $U\left(K^{*}\right)=0$ and $0<K^{*}<\frac{1-\eta}{\eta} \frac{\sigma^{2}}{\gamma P}+1$.

The equality $U\left(K^{*}\right)=0$ is equivalent to $f\left(\operatorname{SIN} R\left(K^{*}\right)\right)=u$, or $\operatorname{SINR}\left(K^{*}\right)=\frac{P N}{\gamma\left(K^{*}-1\right) P+\sigma^{2}}=$ $f^{-1}(u)$. Since the $S I N R$ is a strictly decreasing function of the number of sources, the equation has a (unique) solution if the maximal $S I N R$ when there is only one user $\operatorname{SINR}(1)$ is above $f^{-1}(u)$ and the SINR at capacity constraint is under $f^{-1}(u)$. The first condition defines the maximal price $u_{\max }=f(\operatorname{SINR}(1))=f\left(N P / \sigma^{2}\right)$ above which no user will enter $\left(K^{*}=0\right)$ the system, since his utility will always be negative. The second (capacity) condition defines a price $f\left(\operatorname{SIN} R\left(\frac{1-\eta}{\eta} \frac{\sigma^{2}}{\gamma P}+\right.\right.$ $1))=f\left(\frac{N P \eta}{\sigma^{2}}\right)$ above which the number of sources is less than what capacity allows. If this is not verified, If the price is higher than this threshold, capacity is reached, leading to the third equilibrium case described in the theorem (new customers are not allowed to enter ven of their utility is positive).

If $f\left(\frac{N P \eta}{\sigma^{2}}\right)<u<u_{\max }$, the number $K^{*}$ of sources satisfies $f\left(S I N R\left(K^{*}\right)\right)=u$, that is $K^{*}=1-\frac{\sigma^{2}}{\gamma P}-\frac{N}{\gamma f^{-1}(u)}$.

PI ${ }^{\circ} 1731$ 


\section{B Proof of Theorem 2}

The base station revenue is

$$
R(u, P)=u K^{*}(u, P) \mu(u, P) .
$$

From Theorem 1, we have 3 subdomains for the expression of $K^{*}$ in terms of $(u, P)$ (as illustrated in Figure 7).

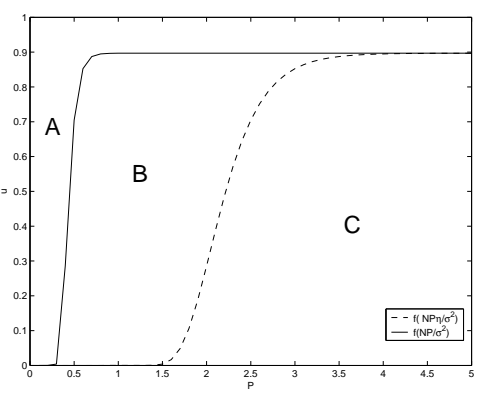

Figure 7: Three equilibrium domains ("nobody" in area A, "full capacity" in area C, or "null utility" in area B) for the number of active users as a function of price $u$ and power $P$.

- In the region of $(u, p)$ such that $f\left(\frac{N P \eta}{\sigma^{2}}\right)<u<f\left(\frac{N P}{\sigma^{2}}\right)$ (where users get null utility), $K^{*}(u, P)=1-\frac{\sigma^{2}}{\gamma P}-\frac{N}{\gamma f^{-1}(u)}$ from Theorem 1. Inserting this in the expression of the average throughput (9) we get

$$
R(u, P)=u\left(1-\frac{\sigma^{2}}{\gamma P}-\frac{N}{\gamma f^{-1}(u)}\right) \frac{r R_{c}}{N}\left(1-e^{-\beta f^{-1}(u)}\right)^{L r}
$$

This function is continuous and differentiable in both of its variables and it is easy to check that $\forall u, P$

$$
\frac{\partial R}{\partial P}(u, P)=\frac{u \sigma^{2}}{\gamma P^{2}}>0 .
$$

Therefore, the revenue over the domain $f\left(\frac{N P \eta}{\sigma^{2}}\right)<u<f\left(\frac{N P}{\sigma^{2}}\right) \Longleftrightarrow \frac{\sigma^{2}}{N} f^{-1}(u)<P<$ $\frac{\sigma^{2}}{N \eta} f^{-1}(u)$ is such that $P=\frac{\sigma^{2}}{N \eta} f^{-1}(u)$, i.e., $u=f\left(\frac{N P \eta}{\sigma^{2}}\right)$, meaning that price and power are configured such that full capacity is reached.

- In the region such that $f\left(\frac{N P \eta}{\sigma^{2}}\right)>u$ (full capacity reached and positive utility meaning that no other user is allowed to enter), Theorem 1 gives $K^{*}(u, P)=1+\frac{1-\eta}{\eta} \frac{\sigma^{2}}{\gamma P}$ leading to the expression of the revenue:

$$
R(u, P)=u\left(1+\frac{1-\eta}{\eta} \frac{\sigma^{2}}{\gamma P}\right) \frac{r R_{c}}{N}\left(1-e^{-\beta \frac{P N}{\sigma^{2}} \eta}\right)^{L r} .
$$


This function is also continuous and differentiable such that

$$
\frac{\partial R}{\partial u}(u, P)=\left(1+\frac{1-\eta}{\eta} \frac{\sigma^{2}}{\gamma P}\right) \frac{r R_{c}}{N}\left(1-e^{-\beta \frac{P N}{\sigma^{2}} \eta}\right)^{L r}>0 .
$$

Again, a maximum over this region is thus necessarily at a point $(u, P)$ such that

$$
u=f\left(\frac{N P \eta}{\sigma^{2}}\right)
$$

on the border such that the utility is zero.

- Over the third region $u>u_{\max }=f\left(\frac{N P}{\sigma^{2}}\right), K^{*}=0$ leading to no revenue.

From what is above, the maximum is necessary on the curve of maximal capacity $u=f\left(\frac{N P \eta}{\sigma^{2}}\right)$, where the revenue is expressed in terms of $P$ by

$$
R(P)=R\left(f\left(\frac{N P \eta}{\sigma^{2}}\right), P\right)=f\left(\frac{N P \eta}{\sigma^{2}}\right)\left(1+\frac{1-\eta}{\eta} \frac{\sigma^{2}}{\gamma P}\right) \frac{r R_{c}}{N}\left(1-e^{-\beta \frac{P N}{\sigma^{2}} \eta}\right)^{L r} .
$$

This function is continuous and differentiable over $[0,+\infty)$. Defining the variable $X=\frac{N P}{\sigma^{2}} \eta$, the revenue can be rewritten as

$$
\begin{array}{r}
R_{1}(X)=R(P)=\frac{r R_{c}}{N}\left(1-e^{-\beta X}\right)^{L r} f(X)+ \\
\frac{1-\eta}{\gamma} r R_{c} \frac{\left(1-e^{-\beta X}\right)^{L r} f(X)}{X} .
\end{array}
$$

Equation $\frac{\partial R}{\partial P}(P)=0$ is equivalent to $\frac{\partial R_{1}}{\partial X}(X)=0$ because $\frac{\partial X}{\partial P}=\frac{N}{\sigma^{2}} \eta \neq 0$. Also,

$$
\begin{aligned}
\frac{\partial R_{1}}{\partial X}(X)= & \frac{r R_{c}}{N}\left(\operatorname{Lr} \beta e^{-\beta X}\left(1-e^{-\beta X}\right)^{L r-1} f(X)+\right. \\
& \left.\left(1-e^{-\beta X}\right)^{L r} f^{\prime}(X)\right)+\frac{1-\eta}{\gamma} r R_{c} \times \\
& \frac{L r \beta X\left(1-e^{-\beta X}\right)^{L r-1} f(X)}{X^{2}}+ \\
& \frac{X\left(1-e^{-\beta X}\right)^{L r} f^{\prime}(X)}{X^{2}}-\frac{\left(1-e^{-\beta X}\right)^{L r} f(X)}{X^{2}} .
\end{aligned}
$$

After some computations, $\frac{\partial R_{1}}{\partial X}(X)=0$ if and only if

$$
\begin{gathered}
f(X)\left(\gamma X^{2} \operatorname{Lr} \beta e^{-\beta X}+X N(1-\eta) \operatorname{Lr} \beta-N\left(1-e^{-\beta X}\right)\right) \\
+f^{\prime}(X)\left(\gamma X^{2}\left(1-e^{-\beta X}\right)+X N\left(1-e^{-\beta X}\right)\right)=0 .
\end{gathered}
$$

Denoting by $\mathcal{X}$ the set of solutions, we obtain prove the theorem.

PI ${ }^{\circ} 1731$ 


\section{Proof of Corollary 1}

The following lemma will be helpful to prove the corollary.

Lemma 1 Let $a$ and $b$ be real numbers such that $a>0$ and $b>1$. The equation

$$
a X^{2}+b X+1=e^{X}
$$

has a unique strictly positive solution.

Proof Define $F(X)=a X^{2}+b X+1-e^{X}$. We have $F(0)=0, \lim _{X \rightarrow \infty} F(X)=-\infty$, $F^{\prime}(X)=2 a X+b$ with $F^{\prime}(0)=b-1>0$, and $F^{\prime \prime}(X)=2 a-e^{X}$.

- If $2 a<1, F^{\prime}$ is strictly decreasing over $[0, \infty)$ (from $F^{\prime \prime}<0$ ). Since $F^{\prime}(0)>0$, there is only one strictly positive solution to the equation.

- If $2 a \geq 1, F^{\prime}$ is first increasing, with $F^{\prime}(0)>0$, then strictly decreasing to $-\infty$ when $X \rightarrow \infty$. Thus $F$ is first increasing and positive (since $F(0)=0$ ), then decreasing to $-\infty$, meaning that there is a unique solution to $F(X)=0$.

The result follows.

We can then prove the corollary.

Proof of Corollary 1. Using the specific function $f$ corresponding to delay, (10) becomes,

$$
\operatorname{Lr}(1+\alpha) \beta X^{2}+\frac{1-\eta}{\gamma} \operatorname{Lr}(1+\alpha) \beta N X+(1-\eta) \frac{N}{\gamma}=\frac{1-\eta}{\gamma} N e^{\beta X} .
$$

This can be rewritten, with $Y=\beta X$ as

$$
a Y^{2}+b Y+1=e^{Y}
$$

where $a=\operatorname{Lr}(1+\alpha) \frac{\gamma}{(1-\eta) N \beta}$ and $b=\operatorname{Lr}(1+\alpha)$. We have $a>0$ and $b>1$ because $b=$ $\operatorname{Lr}(1+\alpha)>\operatorname{Lr}$ and since $L r$, the number of information bytes per packet is typically more than 1 in communication systems. From Lemma 1, (20) has a unique solution $X^{*}$ over $X>0$. Then, Theorem 2 gives the optimal per-packet price

$$
u^{*}=\left(\frac{R_{c}}{L N}\right)^{\alpha}\left(1-e^{-\beta X^{*}}\right)^{\alpha L r},
$$

and the optimal received power is $P^{*}=\frac{X^{*} \sigma^{2}}{N \eta}$. 


\section{Proof of Theorem 3}

The per-packet price and the received power are fixed to $u$ and $P$. Assume that, at a given time, the potential number of users is $\delta$. There are several situations:

- If $\delta=0$, there is no demand, so that the actual number of users $\kappa^{*}$ is $\kappa^{*}=0$.

- If $\delta>K^{*}$ ( $K^{*}$ being the equilibrium value when demand exceeds capacity), assume that there are already $K$ customers in communication. If $K<K^{*}$ (resp. $K^{*}<K \leq \delta$ ), the number of active sessions increases (resp. decreases) exactly in the same way as in the case where demand exceeds capacity (see the proof of Theorem A) since users have a positive (resp. negative) utility, so that finally $\kappa^{*}=K^{*}$.

- If $\delta \leq K^{*}$, all users, up to full demand $\delta$ are served and have positive utility. This means that they all ask for service. Thus, $\kappa^{*}=\delta$.

This proves the theorem.

\section{E Proof of Theorem 4}

The proof studies different cases.

1. If $U_{v}(1,0) \leq 0 \Leftrightarrow u_{v} \geq f_{v}\left(\frac{N_{v} P_{v}}{\sigma^{2}}\right)$ and $U_{d}(0,1) \leq 0 \Leftrightarrow u_{d} \geq f_{d}\left(\frac{N_{d} P_{d}}{\sigma^{2}}\right)$, no user has interest in requesting service when the base station is idle since an entering user will get a negative utility. Additionally, knowing that $U_{d}$ and $U_{v}$ are decreasing functions in both of their variables $K_{v}$ and $K_{d}$, this result also holds for all couple $\left(K_{v}, K_{d}\right)$. Therefore,

$$
\left(K_{v}^{*}, K_{d}^{*}\right)=(0,0) .
$$

2. If $U_{v}(1,0) \leq 0 \Leftrightarrow u_{v} \geq f_{v}\left(\frac{N_{v} P_{v}}{\sigma^{2}}\right)$ but $U_{d}(0,1)>0$, only type- $d$ users apply for service, since $U_{v}\left(K_{v}, K_{v}\right) \leq 0 \forall\left(K_{v}, K_{d}\right)$. As a consequence, we are in the case of a single class studied in Theorem 1, leading to the result.

3. If $U_{d}(0,1) \leq 0 \Leftrightarrow u_{d} \geq f_{d}\left(\frac{N_{d} P_{d}}{\sigma^{2}}\right)$ but $U_{v}(1,0)>0$, we follow exactly the same line of argument, switching type- $v$ and type- $d$.

4. If $U_{v}(1,0)>0$ and $U_{d}(0,1)>0$, users of both types can apply for service.

The Capacity constraint (12) can be rewritten as the linear relation between the number of users

$$
K_{v} P_{v}+K_{d} P_{d}=\frac{1-\eta}{\eta} \frac{\sigma^{2}}{\gamma}+P_{v}+\left(P_{d}-P_{v}\right) \mathbb{1}_{P_{d}<P_{v}} .
$$

Note also that relations $U_{d}\left(K_{v}, K_{d}\right)=0$ and $U_{v}\left(K_{v}, K_{d}\right)=0$ can be rewritten

$$
U_{d}\left(K_{v}, K_{d}\right)=0 \text { iff } K_{v} P_{v}+K_{d} P_{d}=\frac{N_{d} P_{d}}{\gamma f_{d}^{-1}\left(u_{d}\right)}-\frac{\sigma^{2}}{\gamma}+P_{d}
$$

PI ${ }^{\circ} 1731$ 
and

$$
U_{v}\left(K_{v}, K_{d}\right)=0 \text { iff } K_{v} P_{v}+K_{d} P_{d}=\frac{N_{v} P_{v}}{\gamma f_{v}^{-1}\left(u_{v}\right)}-\frac{\sigma^{2}}{\gamma}+P_{v} .
$$

These equations define three parallel lines. The equilibrium depends on the ordering of those lines.

(a) If $\frac{\sigma^{2}}{\eta \gamma}+P_{v}+\left(P_{d}-P_{v}\right) \mathbb{1}_{P_{d}<P_{v}} \leq \min \left(\frac{N_{d} P_{d}}{\gamma f_{d}^{-1}\left(u_{d}\right)}+P_{d}, \frac{N_{v} P_{v}}{\gamma f_{v}^{-1}\left(u_{v}\right)}+P_{v}\right)$, the capacity constraint (12) defines the lowest curve (case (a) of Figure 8). Thus, users apply for service until capacity is reached, so that

$$
K_{v} P_{v}+K_{d} P_{d}=\frac{1-\eta}{\eta} \frac{\sigma^{2}}{\gamma}+P_{v}+\left(P_{d}-P_{v}\right) \mathbb{1}_{P_{d}<P_{v}} .
$$

The base station decides which users to accept and which users to reject. We follow here the policy that the base station chooses users that will result in a larger revenue. The revenue is

$K_{v} \mu_{v} u_{v}+K_{d} \mu_{d} u_{d}=K_{d}\left(u_{d} \mu_{d}-u_{v} \mu_{v} \frac{P_{d}}{P_{v}}\right)+u_{v} \mu_{v}\left(\frac{1-\eta}{\eta} \frac{\sigma^{2}}{\gamma P_{v}}+1+\frac{P_{d}-P_{v}}{P_{v}} \mathbb{1}_{P_{d}<P_{v}}\right)$

using relation (21), where the throughputs $\mu_{v}$ and $\mu_{d}$ are

$$
\mu_{v}^{c}=\frac{r R_{c}}{N_{v}}\left(1-e^{-\beta \frac{N_{v} P_{v}}{\sigma^{2} / \eta+\gamma\left(P_{d}-P_{v}\right) 1_{P_{d}}<P_{v}}}\right)^{L r}
$$

and

$$
\mu_{d}^{c}=\frac{r R_{c}}{N_{d}}\left(1-e^{-\beta \frac{N_{d} P_{d}}{\sigma^{2} / \eta+\gamma\left(P_{v}-P_{d}\right) \mathbf{1}_{P_{v}<P_{d}}}}\right)^{L r},
$$

and they do not depend on $K_{d}$ and $K_{v}$. The revenue is thus linear in $K_{d}$ and the optimal value depends on the sign of $u_{d} \mu_{d}^{c}-u_{v} \mu_{v}^{c} \frac{P_{d}}{P_{v}}$. This provides the result.

(b) If $\frac{N_{d} P_{d}}{\gamma f_{d}^{-1}\left(u_{d}\right)}+P_{d}<\min \left(\frac{\sigma^{2}}{\eta \gamma}+P_{v}+\left(P_{d}-P_{v}\right) \mathbb{1}_{P_{d}<P_{v}}, \frac{N_{v} P_{v}}{\gamma f_{v}^{-1}\left(u_{v}\right)}+P_{v}\right)$, the curve $U_{d}\left(K_{v}, K_{d}\right)=0$ is the lowest one. (cases (d) and (e) of Figure 8). Then users enter the system until this curve is reached. Yet, type- $v$ users have positive utility, so they continue to enter. At the same time, the utility of type- $d$ users becomes negative so that some of them leave. We therefore slide on the curve $U_{d}\left(K_{v}, K_{d}\right)=0$ until $K_{d}=0$. Then $K_{v}$ still increases until capacity is reached (case (d) of Figure 8) or $U_{v}\left(K_{v}, K_{d}\right)=0$ (case (e) of Figure 8).

The equilibrium is thus $\left(K_{v}^{*}, 0\right)$ with $K_{v}^{*}$ defined in (14).

(c) Else if $\frac{N_{v} P_{v}}{\gamma f_{v}^{-1}\left(u_{v}\right)}+P_{v}<\min \left(\frac{N_{d} P_{d}}{\gamma f_{d}^{-1}\left(u_{d}\right)}+P_{d}, \frac{\sigma^{2}}{\eta \gamma}+P_{v}+\left(P_{d}-P_{v}\right) \mathbb{1}_{P_{d}<P_{v}}\right)$, the result follows by a similar argument, switching just type- $v$ and type- $d$ (cases (b) and (c) of Figure 8).

This concludes the proof. 


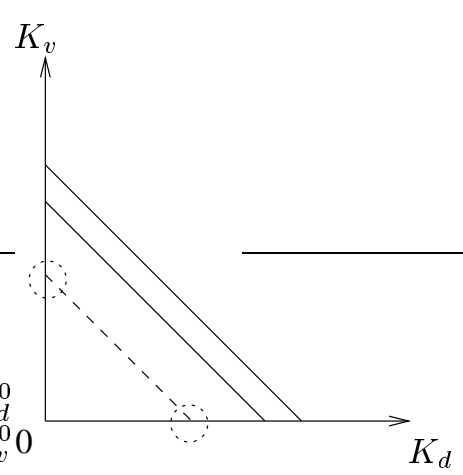

(a)

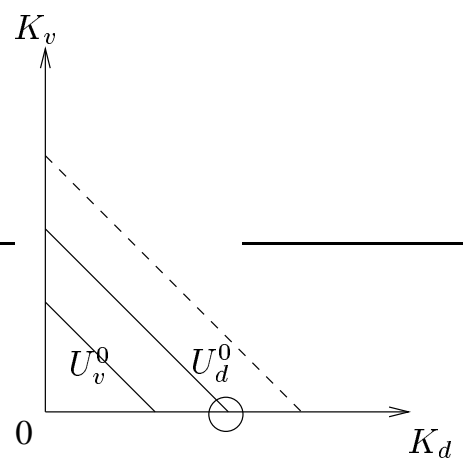

(c)

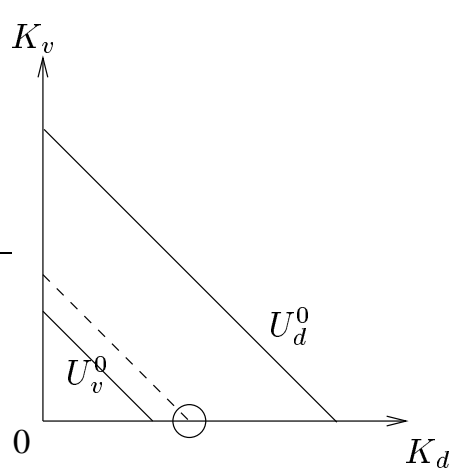

(b)

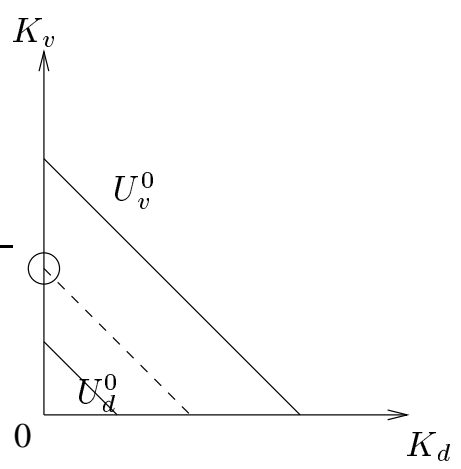

(d)

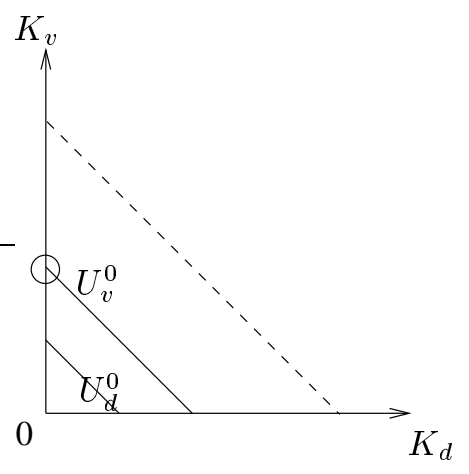

(e)

Figure 8: Equilibrium depending on prices and received powers in the case where demand exceeds capacity. The dashed line represents the capacity constraint. Curve $U_{v}^{0}$ is for $U_{v}\left(K_{v}, K_{d}\right)=0$ while curve $U_{d}^{0}$ is for $U_{d}\left(K_{v}, K_{d}\right)=0$. Circled points indicate the Nash equilibria. PI n ${ }^{\circ} 173 l^{d}$ 


\section{F Proof of Theorem 5}

Following Theorem 4, there is only one type of user at equilibrium. Thus we shall choose the type $i \in\{v, d\}$ so $R_{i}^{*}=\max \left(R^{*} v, R_{d}^{*}\right)$. From Theorem 2 for the case of a single class, this is given by

$$
u_{i}^{*}=f_{i}\left(X^{*}\right) \quad \text { and } \quad P_{i}^{*}=\frac{X^{*} \sigma^{2}}{N_{i} \eta},
$$

with $X^{*}$ defined by:

$$
X^{*}=\arg \max _{X \in \mathcal{X}} \frac{r R_{c}}{N}\left(1-e^{-\beta X}\right)^{L r} f_{i}(X)+\frac{1-\eta}{\gamma} r R_{c} \frac{\left(1-e^{-\beta X}\right)^{L r} f_{i}(X)}{X} .
$$

Though, the values of $u_{j}^{*}$ and $P_{j}^{*}$ with $j \in\{v, d\}, j \neq i$, are chosen from Theorem 4 so that only class $-i$ users are present at equilibrium. This is provided by the range of values given in the theorem. 